\title{
GOBIERNOS CORPORATIVOS Y EL FRAUDE TRIBUTARIO: UN ANÁLISIS TEÓRICO Y DOS ESTUDIOS DE CASOS
}

\section{CORPORATE GOVERNANCE AND TAX FRAUD: A THEORETICAL ANALYSIS AND TWO CASE STUDIES}

\author{
Claudia Orellana Fuentes ${ }^{1}$ y Digna Azúa Álvarez ${ }^{2}$
}

\section{RESUMEN}

Los ingresos del Estado obtenidos vía impuestos son considerados uno de los principales recursos para la lucha contra la pobreza. Este estudio busca establecer la relación entre la definición de Gobierno Corporativo (GC) y los impuestos. Los impuestos pueden ser considerados como la retribución justa al capital social aportado por el Estado, o un simple gasto que debe ser reducido al máximo. Así, en una primera parte, se analiza la acepción utilizada en Chile respecto de los impuestos, la evidencia sugiere que la Sociedad chilena considera que el GC solo crea valor para el accionista y en consecuencia de ello, el impuesto es una transferencia de valor desde el accionista hacia el Estado. Además, se analizan el concepto de fraude.

La segunda parte comprende dos estudios de casos sobre posibles fraudes tributarios, el Grupo Penta, financiado con capitales privados y Codelco-Minmetals, una empresa estatal. En los estudios de caso se presentan los antecedentes sobre la investigación de los hechos, para luego exponer los GC de dichas empresas con el fin de establecer una relación. En ambos casos se ha detectado una disfunción en los GC que propicia la ocurrencia de situaciones de fraude fiscal, lo cual se ve potenciado por la presión para la obtención de mayores beneficios en el caso de Penta, o por la búsqueda de financiamiento para nuevos proyectos en el caso de Codelco. El uso de los precios de transferencia y la deslocalización de resultados hacia paraísos fiscales cuestiona el rol de la contabilidad, que no es neutro, en la distribución de ingresos y su impacto en la desigualdad social y la disminución de la pobreza.

Palabras claves: Fraude fiscal, gobierno corporativo, teoría de la agencia, teoría de las partes interesadas.

Recepción: 06/06/2016. Aprobación: 29/06//2016.

\begin{abstract}
Taxes are considered one of the main resources for the fight against poverty. This study seeks to establish the relationship between the definition of Corporate Governance (CG) and taxes. Taxes can be seen as the just retribution to State social capital, otherwise, as simple expenses to be reduced to a minimum. Thus, in a first part, this text shows the analysis of the tax's meaning being used in Chile. The evidence suggests that the Chilean Society believes that the CG only creates shareholder value. As a consequence, the tax is a transfer of value from shareholder to State. In addition, the paper analyzes tax fraud conceptualization.
\end{abstract}

1 Escuela de Comercio, Facultad Económica y Administrativa, Pontificia Universidad Católica de Valparaíso, Chile, claudia.orellana@ucv.cl 2 Escuela de Comercio, Facultad Económica y Administrativa, Pontificia Universidad Católica de Valparaíso, Chile, digna.azua@ucv.cl 
The second part comprises of two studies. A possible Penta Group's tax fraud financed by private capital and Codelco-Minmetals, a state based company. In these case studies, it is presented the facts and the history of the investigation. Then, the authors establish a relationship between CG and the facts. In both cases the study has detected a malfunction in the CG that favors the occurrence of situations of tax fraud. In the case of Penta, the relationship is enhanced by the pressure for greater benefits. Likewise, Codelco-Minmetals relationship is based on the need to finance new projects. The use of transfer pricing and the relocation of results to taxes paradises put into question the role of accounting. Accounting is not neutral, and fulfills an important role in the distribution of income and its impact on social inequality and, consequently, into poverty reduction.

Keywords: Tax fraud, corporate governance, agency theory, stakeholder's theory.

\section{INTRODUCCIÓN}

El Ministerio de Hacienda de la República de Chile ${ }^{3}$ señala que "Los impuestos son cobrados para que el gobierno pueda cumplir con una serie de objetivos": i) Corregir externalidades, esto es disponer de los recursos para financiar gasto público ocasionado por ciertas actividades económicas; ii) Proveer Bienes Públicos, tales como gastos en defensa, salud educación; y iii) Redistribuir riqueza. Dada la importancia de este rol, diversas organizaciones no gubernamentales a nivel mundial han tomado como estandarte la lucha contra la corrupción y la evasión fiscal, iniciativas como Publish What You Pay (PWYP), Extractive Industries Transparency Initiative (EITI), ATTAC, Oxfam, Action Aid, Tax Justice Network, Financial Transparency Coalition (FTC), entre muchos otros que luchan contra los paraísos fiscales y la evasión fiscal. No obstante, nuevos escándalos de fraude fiscal siguen ocupando un lugar importante en la prensa nacional e internacional.

Para los países, la evasión fiscal tiene consecuencias nefastas, prueba de ello es la crisis económica que enfrenta Grecia, en donde "el fraude y la evasión fiscal alcanzó y alcanza aún niveles altísimos que explican en gran parte la crisis griega: El FMI y la Comisión [Europea] la estimaban, en el 2012, entre 40 y 45 billones por año, es decir entre el 12 y $15 \%$ del PIB" ${ }^{\prime \prime}$ (trad. propia).

Esta evidencia sugiere, entonces, que la evasión fiscal parece ser una actividad económica trivial, en opinión de la Association of Certified Fraud Examiners "el fraude es omnipresente; no discrimina en su ocurrencia. Y mientras que los controles antifraudes pueden reducir efectivamente la probabilidad y el impacto potencial de fraude, lo cierto es que ninguna entidad es inmune a esta amenaza" (ACFE, 2014, p. 6, trad. propia).

Respecto de Latinoamérica, organismos como Oxfam (2014, p. 1) han declarado que "la recaudación tributaria en Latinoamérica y el Caribe es baja en relación con su potencial y no se corresponde con las inmensas necesidades sociales de la región. Las finanzas públicas reflejan un modelo social y económico inequitativo que perpetúa la concentración del poder y las discriminaciones, y están socavadas por los altos indices de evasión y elusión fiscal. Urgen reformas valientes para lograr políticas fiscales que ayuden a reducir las desigualdades enquistadas en la región".

Jiménez et al. (2010, p. 7) declaran que "los países de América Latina tributan poco y mal. Se caracterizan por tener una baja presión tributaria, una estructura sesgada hacia impuestos regresivos y tasas de evasión y elusión fiscal bastante elevadas, lo que restringe la posibilidad de instrumentar politicas fiscales redistributivas y su efectividad".

Atendiendo a lo anteriormente expuesto, este estudio tiene como objetivo analizar el fraude tributario desde la perspectiva de los Gobiernos Corporativos, bajo el prisma de la línea de investigación en contabilidad crítica que reconoce los juegos de actores en las diversas construcciones sociales que resultan de los procesos de negociación tales como las normas contables, las leyes tributarias, etc., en un momento y lugar determinado (Capron, 2005; Richard, 2009; Richard, Collette, Bensandon \& Jaudet, 2011).

La metodología de trabajo considera abordar el análisis desde un enfoque teórico y un enfoque práctico. Por una parte, el enfoque teórico considera asociar el rol de los impuestos con dos

3 Ministerio de Hacienda, (2015, 07, 31) www.hacienda.cl. [on line]. Disponible en: http://www.hacienda.cl/preguntas-frecuentes/impuestos/porque-los-ciudadanos-tienen-que-pagar.html

4 Catremer, J. (2014, 01, 03) En Grèce, l'impôt n'est toujours pas pour tous [on line]. Disponible en : http://bruxelles.blogs.liberation.fr/2014/01/03/engrece-limpot-nest-toujours-pas-pour-tous / (31/07/2015). 
conceptos distintos de Gobiernos Corporativos (GC), conocidos como el concepto tradicional ${ }^{5}$ y el concepto pluralista ${ }^{6}$. Estas nociones de GC serán confrontadas con la concepción del sistema tributario existente en Chile. De esta forma se podrá establecer que acepción de GC vinculada al desarrollo del sistema tributario de un país, podría contribuir a disminuir el fraude tributario.

Por otra parte, el enfoque práctico tendrá por finalidad, presentar dos estudios de casos de evasión y / o elusión fiscal, uno que involucra una empresa estatal de renombre como CODELCO, y otro relativo a uno de los Grupos Financieros más importantes del país, el Holding Penta. El propósito de este análisis es constatar si existe vinculación entre los hechos acontecidos y el GC de cada organización.

\section{ENFOQUE TEÓRICO}

A partir del postulado de que la medición tradicional de la generación de valor para los accionistas, es demasiado restrictiva para la construcción de una teoría de GC para las empresas, Charreaux \& Desbrières (1998) plantean las bases de una definición ampliada del valor creado, el cual es llamado el valor partenarial, que propone una visión pluralista de la firma, la cual considera el conjunto de Stakeholders que participan en el funcionamiento de ésta.

En concordancia con lo anterior, la concepción del impuesto es reconocida de forma diametralmente distinta en estas dos visiones. De esta forma, en el contexto de un enfoque tradicional de GC el impuesto es considerado una carga tributaria, un gasto que merma el retorno para el accionista y disminuye el valor que la empresa genera, "esta ideología retrata el impuesto como una transferencia de los accionistas al Estado en lugar de un retorno a la sociedad por la inversión de capital social y por lo tanto se refiere a la evasión de los impuestos como una práctica comercial normal y de sentido común" (Sikka, 2010, p. 156, trad. propia).

Por lo tanto, existe una constante confrontación entre el cumplimiento de la obligación fiscal y los beneficios que esperan recibir los accionistas, "la búsqueda de beneficios requiere que los directores equilibren los intereses de los diversos grupos de interés, incluyendo la obligación de pagar impuestos al Estado y la Sociedad. El uso de estrategias de elusión/evasión de impuestos es ante todo una cuestión de discreción ejecutiva en lugar de cualquier obligación legal o moral" (Sikka, 2010, p. 155-156, trad. propia).

Por el contrario, en el contexto de un enfoque pluralista de GC el impuesto no es considerado una carga que disminuye el valor creado para el accionista, pues en este modelo el Estado es un Stakeholders por derecho, que permite el ejercicio de la actividad en el territorio y proporciona todos los servicios básicos y bienes públicos para que ésta opere normalmente ${ }^{7}$.

En este contexto, y conforme a la evidencia obtenida en estudios previos, constatamos que el concepto de GC que prevalece tanto a nivel teórico como a nivel práctico en Chile, responde a un concepto tradicional fundado en la Teoría de la Agencia cuyo objetivo fundamental tiene relación con la creación y la protección del valor para los accionistas (Orellana \& Azúa, 2014). Este modelo de GC ha sido importado de Instituciones Internacionales ${ }^{8}$ que promueven esta visión con el fin de convertir a países tales como Chile, en países atractivos para la inversión extranjera materializándose en la instalación de las grandes empresas multinacionales.

Sin embargo, es necesario constatar que una visión pluralista de GC, puede adaptarse mejor a la realidad económica chilena, en donde son múltiples los stakeholders que pueden verse afectados por las actividades de las empresas y las decisiones de sus altos ejecutivos y directorios; agregando además que la mayoría de los grandes grupos económicos del país operan como empresas familiares y no como sociedades anónimas abiertas.

De esta forma, si Chile está utilizando un concepto tradicional de GC, se explica entonces, que los contribuyentes chilenos perciban a los impuestos como una carga que debe ser evitada, utilizando diversos mecanismos. Según Rivas y Paillacar (2007, p. 20-21) estos son: la planificación tributaria, la elusión tributaria y la evasión tributaria. Según estos autores la planificación tributaria no tendría un carácter doloso, sin embargo, la elusión y evasión supone una conducta dolosa que puede realizarse a través del "frau-

5 Basada en la Teoría de la Agencia y la Teoría de shareholders. 6 Basada en la Teoría de los Stakeholders.

7 "El Estado en nombre de la sociedad proporciona capital social en la forma de la educación, la sanidad, el transporte, la seguridad, el sistema legal, los subsidios y el apoyo a las empresas, y los bienes públicos. Cada capital espera recibir la necesaria rentabilidad de su inversión" (Sikka, 2010, p. 155, trad. propia).

8 Estas Instituciones Internacionales son: OCDE, Banco Mundial, FMI, etc. 
de a la ley" (p. 22). Por otra parte, Yañez (2015) plantea que los mecanismos para disminuir el pago de impuestos son: la elusión y la evasión. El autor señala que la elusión es un concepto empleado en finanzas públicas desde dos sentidos, el primero corresponde al "uso de beneficios que presentan los impuestos en sus textos legales, por lo tanto, [...] es un acto lícito" (p. 253), el segundo se "trataría de una acción que no estaría de acuerdo con el espiritu de la ley" (p. 254), en este caso los contribuyentes caen en conductas ilícitas. Respecto de la evasión, se indica que es una acción ilícita o ilegal.

Podemos concluir que existe un área difusa que es llamada por Rivas y Paillacar (2007) como planificación tributaria, y por Yánez (2015) como elusión en su acepción lícita. Estas propuestas corroboran lo expuesto previamente, en donde existe en Chile la percepción de que los impuestos son una carga que debe ser evitada utilizando mecanismos que en ocasiones limitan con conductas ilícitas tales como el fraude.

Respecto a la definición de fraude tributario, en los textos legales consultados, tales como el Código Tributario y el Código Procesal Penal, no se encontraron definiciones explícitas de éste. Es por ello, que evocamos las definiciones desarrolladas por diversos autores chilenos, entre ellos Jorratt (2013) y Van Weezel (2007), los cuales coinciden en que existen diversas configuraciones de fraude tributario. De acuerdo a Jorratt (2013, p. 49) es necesario distinguir distintos términos que en el lenguaje común suelen confundirse relativos al fraude tributario. El primer concepto se conoce como "brecha tributaria" que constituye "la diferencia entre lo que el gobierno debería recaudar, de acuerdo con la letra y el espiritu de la legislación tributaria, y lo que realmente recauda". Jorratt detecta tres orígenes de la brecha tributaria: "subdeclaración involuntaria, elusión tributaria y evasión tributaria". La primera categoría es producto de "errores involuntarios que puede cometer un contribuyente al momento de preparar su declaración de impuestos", atribuidos a la ignorancia del contribuyente. Para este autor, la elusión tributaria "es un concepto algo etéreo, que hace referencia al uso abusivo de la legislación tributaria, es decir, que no respeta el espiritu de la ley, con el propósito de reducir el pago de impuestos", y la evasión tributaria, que consiste en "la subdeclaración ilegal y voluntaria de los impuestos. En este caso hay un acto deliberado por parte del contribuyente para reducir sus obligaciones tributarias. Dependiendo de la gravedad de estos actos, podrán ser tipificados como faltas o delitos tributarios".

Este estudio está abocado a exponer dos casos que están siendo investigados porque pueden constituir fraude tributario. Uno de ellos está referido a la empresa estatal más importante del país: CODELCO.

Tanzi (2001) expuso lo que él determinó como las termitas fiscales las que merman "en la capacidad de los países para obtener ingresos a través de su sistema tributario" (p. 34), tanto el abuso en la fijación de los precios de transferencia entre filiales, en donde expuso que "hay indicios de que algunas empresas manipulan los precios para trasladar utilidades de jurisdicciones con impuestos muy elevados a otras con impuestos bajos" (p. 35), como la existencia de centros financieros extraterritoriales o "paraísos fiscales" son consideradas como termitas fiscales producto de una globalización creciente. De acuerdo a Sikka \& Willmott (2010, p. 352, trad. propia) "los precios de transferencia no es sólo una técnica contable, sino también un método de asignación de recursos y la evasión de impuestos que afecta a la distribución de los ingresos, la rique$z a$, los riesgos y la calidad de vida" (p. 352). Sikka (2010, p. 153, trad. propia) declara que pese al gran número de literatura nueva en materia de responsabilidad social "se ha prestado poca atención a la evasión fiscal organizada por las empresas a pesar de que tiene consecuencias reales para las oportunidades de vida de millones de personas".

De acuerdo al estudio de Jorratt (2013) ${ }^{9}$, la evasión fiscal en Chile para el Impuesto al Valor Agregado, entre los años 2008-2011 varió entre el 12 y $18 \%$, el autor estima que para "cada punto de evasión en el IVA significa una pérdida de recaudación de unos US\$200 millones anuales, lo que equivale a 0,1\% del PIB" (p. 104). Para el caso de la evasión del Impuesto de Primera Categoría, entre los años 2005-2009 la tasa anual de evasión fluctuó entre 22 al 34\%, en donde "cada punto de evasión en el Impuesto de Primera Categoría significa una pérdida de recaudación de unos US\$94 millones anuales, lo que equivale a $0,05 \%$ del PIB" (p. 104).

En la siguiente sección se exponen dos estudios de caso acerca de investigaciones que están en proceso y que dejan en evidencia que la evasión afectaría transversalmente a nuestra sociedad, viéndose involucrada una empresa privada y una empresa del sector público, Penta y Codelco-Minmetals, respectivamente.

$9 \mathrm{El}$ autor utiliza fuentes provenientes del Servicio de Impuesto Interno (SII) y estimaciones propias en base a la información de las Cuentas Nacionales chilenas. 


\section{ENFOQUE PRÁCTICO}

Se expondrán dos situaciones de posible fraude tributario que se encuentran en proceso de investigación: el "caso Penta" y el "caso Codelco-Minmetals". El uso de la metodología de estudio de casos nos parece pertinente, pues ella "no se basa en una muestra estadística en ningún sentido positivista, porque las empresas rara vez proporcionan información sobre su evasión y estrategias de evasión y, por tanto, el tamaño de las poblaciones apropiadas no se puede determinar de una manera significativa" (Sikka, 2010, p. 157, trad. propia).

La riqueza de este estudio radica en la presentación de la evasión tributaria en empresas chilenas con capitales privados y capitales públicos.

Para ambos casos se presentarán: (1) los aspectos relevantes que están siendo investigados y (2) la estructura de Gobierno Corporativo desarrollado en cada organización, descrito en función de los antecedentes públicos disponibles.

\subsection{Caso Penta}

\subsubsection{Antecedentes del caso Penta}

La génesis de este caso fue una investigación llevada a cabo por el SII, denominada el "Fraude al FUT", "caso en el que los imputados realizaron operaciones que simulaban pérdidas tributarias para obtener devoluciones irregulares de Impuesto a la Renta, por los períodos tributarios 2007 al 2010"10 en una red de corrupción en la cual participaron activamente funcionarios del SII, en donde "el total de querellados por el caso alcanza a 125. De estos, un grupo de 6 contadores, 1 abogado y 2 ex funcionarios de la institución fueron los que conformaron una verdadera red delictual para defraudar al fisco, mientras que el resto corresponde a contribuyentes que obtuvieron maliciosamente devoluciones improcedentes por sumas que van entre los $\$ 6$ y $\$ 113$ millones"11.

Entre los contribuyentes imputados por obtener devoluciones indebidas, se encontraba Hugo Bravo, que en ese entonces era el Presidente del Banco Penta, siendo desvinculado de ésta ipso facto. Hugo Bravo que fue el planificador tributario del Grupo Penta y mano derecho de los socios fundadores, no contento con su salida del Grupo entregó antecedentes al SII sobre los delitos tributarios cometidos con sus antiguos empleadores.

En agosto de 2014, el SII formaliza a los socios fundadores del Grupo Penta, Carlos Délano y Carlos Lavín, por el delito tributario de "la utilización indebida de boletas de honorarios falsas de sus cónyuges para disminuir la base imponible del Impuesto a la Renta de la sociedad Inversiones Penta III Limitada, de la que son sus representantes"12, dicha querella se amplió posteriormente contra los representantes legales de varias filiales del grupo en donde se observaron este tipo de irregularidades. En marzo de 2015, el SII declaraba que "las empresas del grupo incorporaron como gasto en su contabilidad un total de 659 documentos falsos, entre facturas no afectas o exentas de IVA y boletas de honorarios, por un monto total de \$3.302.544.451"13, un revuelo mediático importante tomó este caso cuando se constató que el destino de los dineros obtenidos con esta documentación, no sólo se dirigía a pagar sobresueldos a sus altos ejecutivos o encubrir retiros de sus dueños pero también, encubría donaciones a personalidades políticas, es decir financiamiento irregular de campañas políticas.

En marzo y abril de 2015 se sumaron dos nuevas querellas en contra de los socios fundadores y los representantes legales de las filiales involucradas en la investigación, pero esta vez en torno a un nuevo delito tributario que consistía en "la rebaja indebida de la renta líquida imponible de las compañias y la evasión del Impuesto Único establecido en el artículo 21 de la Ley Sobre Impuesto a la Renta, al registrar como gasto en su contabilidad y luego incorporar en sus declaraciones de impuestos pérdidas ficticias originadas en operaciones de forwards artificiosas ${ }^{\prime 14}$.

Dichas operaciones de forwards consistían en establecer un contrato con un intermediario, fijando una fecha falsa anterior a la verdadera, por lo tanto no tenían la incertidumbre de todo contrato a futuros, pues los tipos de cambio de los dólares ya eran conocidos, el objeto de ello era obtener deliberadamente una pérdida financiera para las empresas del Grupo Penta y, al mismo tiempo, la compañía intermediara realizaba un contrato similar con una empresa perteneciente a la persona a la cual el Grupo Penta deseaba transferir dichos fondos, estos contratos de forwards, antedatados igualmente, se mani-

10 SII, (2014, 07, 25) www.sii.cl. [on line]. Disponible en: http:/ / www.sii.cl/pagina/actualizada/noticias/2014/250714noti01rr.htm (31/07/2015). 11 Op. cit.

12 SII, (2014, 08, 26) www.sii.cl: [on line]. Disponible en: http://www.sii.cl/pagina/actualizada/noticias/2014/260814noti01cs.htm (31/07/2015). 13 SII, (2015, 03, 15) www.sii.cl: [on line]. Disponible en: http://www.sii.cl/pagina/actualizada/noticias/2015/020315noti01jv.htm (31/07/2015). 14 SII, (2015, 04, 23) www.sii.cl: [on line]. Disponible en: http://www.sii.cl/pagina/actualizada/noticias/2015/230415noti01jv.htm (31/07/2015). 
pulaban para generar ganancias en su liquidación. La compañía intermediara a su vez ganaba una comisión por realizar el montaje.

Los contratos de forwards efectuados con las compañías Consultorías y Proyectos y Forestal Valparaíso "se efectuaron entre los años tributarios 2009 a 2013 y generaron un perjuicio fiscal que, de acuerdo a la estimación inicial realizada por fiscalizadores de la Subdirección Jurídica, asciende a más de $\$ 2.400$ millones" $^{\prime 15}$. Los contratos de forwards efectuados con Asesorías VSA Ltda., fueron estimados en 370 millones de pesos ${ }^{16}$.

\subsubsection{Gobierno Corporativo del Grupo Penta}

Un analista de Humphreys en un informe emitido en $2014{ }^{17}$ declaraba que respecto a "las prácticas del gobierno corporativo de Penta Financiero, éste presenta los estándares tradicionales de compañias medianas con elevada concentración propietaria. En este sentido, existe una fuerte presencia de los accionistas en el directorio, sin la figura de directores independientes". En efecto, los socios fundadores del Grupo, Délanos y Lavín, mantienen el control en el patrimonio de la mayoría de las filiales del Holding. Una compleja red de filiales de diferentes naturalezas jurídicas ${ }^{18}$ hace difícil transparentar la información económica y financiera del Grupo y la concentración de la propiedad hace que los Gobiernos Corporativos respondan a una organización similar a las empresas familiares, en donde los dueños tienen plena potestad en la toma de decisiones.

En cuanto al directorio del Banco Penta, Humphreys en su informe emitido en febrero 2014 declaraba que "la administración de la sociedad es ejercida por un directorio elegido por la junta de accionistas y compuesto por siete miembros titulares y dos suplentes. Los directores pueden ser reelegidos en sus cargos por períodos sucesivos de tres años, en forma indefinida"19 situación que se complementa con la presencia de los socios fundadores en la decisiones del directorio.

Actualmente, los socios fundadores y dos altos ejecutivos del Grupo Penta se encuentran formalizados bajo el delito de cohecho, lo cual hace aplicable la Ley 20.393 sobre la Responsabilidad Penal de las Personas Jurídicas, siempre que se pruebe que estos altos ejecutivos no cumplieron sus deberes de dirección y de supervisión: En el caso particular del Grupo Penta, fue implementado un modelo de prevención de delitos debidamente certificado en varias de sus filiales, lo que a priori evitaría la sentencia máxima de la Ley que consistiría en la disolución de la persona jurídica.

Sin embargo, de acuerdo a los hechos imputados a los altos directivos, los modelos de prevención de delitos que declara poseer el Grupo Penta no estarían funcionando correctamente, o simplemente demuestra que son meras declaraciones de intención sin una real implicancia en la toma de decisiones.

\subsection{Caso Codelco-Minmentals}

\subsubsection{Antecedentes del caso Codelco-Minmen- tals}

En el año 2005 Codelco, con Juan Villarzú como Presidente Ejecutivo y el Ministro de Hacienda de la época, Andrés Eyzaguirre que formaba parte integrante del directorio de Codelco, buscaba financiar un nuevo proyecto que dio lugar a la actual Mina Gabriela Mistral. El financiamiento a través de deuda estaba comprometido debido a las restricciones administrativas que tenía Codelco, es así como llegaron a concretar una engorrosa operación con la compañía china Minmetals, "acuerdo [que] fue incomprendido por la opinión pública que no entendía que el objeto final era dar vida al proyecto Gaby [...] de nada sirvió para tranquilizar los ánimos que los asesores financieros fueran UBS y Asset Chile por el lado de Codelco y NM Rothschild secundara a los chinos"20.

La operación consistió en la firma de un acuerdo que involucraba la creación de una filial (Joint Venture) en las Bermudas, Cooper Partners Investment Company Ltd. (CuPIC), cuya participación en el patrimonio se repartió en partes iguales entre las dos sociedades. Además se firmó un contrato de futuro para el suministro de

16 SII, (2015, 03, 02) www.sii.cl: [on line]. Disponible en: http:/ / www.sii.cl/pagina/actualizada/noticias / 2015/020315noti01jv.htm (31/07/2015).

17 Humphreys, (2014, 11) www.humphreys.cl: [on line]. Disponible en: http:/ / www.humphreys.cl/resources/uploads/2014-12/informe-penta-financiero-nov-2014.pdf (31/07/2015).

18 En el Holding se observan principalmente Sociedades Anónimas Cerradas y Sociedades de Responsabilidad Limitada, las cuales quedan fuera de los requerimientos legales de hacer públicos sus Estados Financieros. Actualmente es imposible obtener un Estado Financiero Consolidado del grupo, pues la empresa que consolida en lo alto de la jerarquía es una Sociedad Anónima Cerrada, Empresas Penta S.A.C.

19 Banco Penta, (2014, 02) www.bancopenta.cl: [on line]. Disponible en: http://www.bancopenta.cl/LinkClick.aspx?fileticket=velYX$2 \mathrm{~b} 5 \mathrm{Hg} \% 3 \mathrm{~d} \&$ tabid $=250(31 / 07 / 2015)$.

20 Radic, S. (2013, 12, 15). Aún se sienten los coletazos del acuerdo con Minmetals: Las operaciones de Codelco en Bermudas que no gustaron a Impuestos Internos. [on line] El Mercurio. Disponible en: http:/ / impresa.elmercurio.com/Pages/ NewsDetail.aspx?dt=15-12-2013\%200:00:00\&News$\mathrm{ID}=189516 \& d \mathrm{~dB}=28-09-2015 \%$ 200:00:00\&BodyID=2\&PaginaId=6 $(31 / 17 / 2015)$. 
cobre por 15 años (hasta el año 2021), en donde se fijó una venta anual de 55.750 toneladas de cobre, es decir 836.250 toneladas totales. El precio se determinó de acuerdo a una fórmula con un componente fijo y otro variable, que hacía variar el precio en un rango de US\$1.03-1.05 la libra ${ }^{21}$. Este contrato, consideraba igualmente, la suscripción de un préstamo de CuPIC con la China Development Bank por US $\$ 550$ millones $^{22}$, los cuales fueron remesados a Codelco bajo el concepto de pago anticipado a futuros embarques de cobre, lo que fue utilizado para financiar las inversiones en la Mina Gaby.

El cobre comprado por CuPIC sería revendido a precios corrientes de mercado vigente al momento del embarque a una filial de la minera Minmetals, y los resultados obtenidos por $\mathrm{Cu}$ PIC producto de esta venta, serían repartidos en partes iguales entre Codelco y Minmetals. Codelco, pagaría finalmente impuestos por los dividendos repatriados, pero Minmetals no pagaría nunca impuestos en Chile.

Sin embargo, los precios establecidos en el contrato de futuro resultaron ser muy inferiores al precio de mercado del metal, inclusive inferiores a los costos de producción ${ }^{23}$, lo que produjo grandes pérdidas para Codelco, pérdidas para el Estado chileno, y un gran beneficio para Minmetals. Otra consecuencia de esta operación fue que el Fisco recaudara menos Royalty el cual es calculado sobre el valor de las ventas registradas en Codelco, y a su vez menos Impuestos de Primera Categoría, derivados de las ventas a precios inferiores a los costos de producción, generando de esta forma, la deslocalización de ganancias en las Bermudas vía los precios de transferencia. El SII, aplicando sus facultades de fiscalización frente a los casos de vulneración de los Precios de Transferencia, emitió el 30 de julio de 2010 las liquidaciones de impuestos $\mathrm{N}^{\circ} 1$ y 2 y la Resolución Ex. SDF ํ 1, el 29 de junio de 2012, las liquidaciones de impuestos $\mathrm{N}^{\circ} 45,46$ y $47 \mathrm{y}$ el 27 de diciembre de 2014 las liquidaciones de impuestos $\mathrm{N}^{\circ} 7$ y $8^{24}$. El monto de dichas liquidaciones no fueron hechas públicas ya que Codelco impugnó dichos montos y solicitó la Revisión de la Actuación Fiscalizadora (RAF). De acuerdo a la escueta información entregada en la Memoria Anual de Codelco $2014^{25}$, la Corporación estaría en proceso de negociación con el SII, con el objeto de no llevar el caso a los Tribunales Tributarios. Dicho proceso es considerado de carácter reservado, por lo tanto no existe información de carácter público al respecto.

En junio 2014 se constituyó una Comisión Investigadora de la Cámara de Diputados con el objeto de indagar sobre los contratos de operaciones de futuro y forwards de Codelco contratados entre los años 2005-2007. Dicha Comisión estimó en US\$4.663,30 millones ${ }^{26}$ las pérdidas producto de estos contratos de futuros entre los años 2006-2012, derivadas del acuerdo con Minmentals y recomienda en su informe final "renegociar los términos del mismo para restablecer los equilibrios originales, los que se hicieron trizas un tanto después de firmado el Acuerdo por el vuelco inmenso e inesperado que tuvo el mercado" (p. 168). La Comisión no establece personas responsables y declara que "en la opinión de la mayoría de los integrantes de la Comisión, no se ha acreditado elusión tributaria o perjuicio fiscal" (p. 167).

En consideración a la recomendación de la Comisión Investigadora, el actual Presidente Ejecutivo de Codelco, Nelson Pizarro, declaró "en la conferencia del balance de la cuprera del primer trimestre [...] reconoció que la empresa ha intentado hacer gestiones para cambiar los términos del acuerdo, pero que hasta ahora no ha tenido éxito" 27.

\subsubsection{Gobierno Corporativo de Codelco}

Como uno de las condiciones del ingreso de Chile a la OCDE fue la modernización de los Gobiernos Corporativos de Codelco, el cual se

21 Editorial La Tercera (2014, 06, 26). Codelco-Minmetals: a las dudas se sumó el SII [on line] La Tercera. Disponible en: http://www.latercera.com/ noticia/opinion/editorial/2014/06/894-584033-9-codelcominmetals-a-las-dudas-se-sumo-el-sii.shtml (31/07/2015).

22 "Para llegar a la suma, tanto Codelco como Minmetals aportaron US\$110 millones cada una a CuPIC, completándose el saldo con un crédito que le otorgó Chino Development Bank. Este préstamo fue hecho por un plazo de 11 años a una tasa de interés fija anual de 6.5\%, el cual ya fue completamente pagado". Radic, S. El mercurio $(15 / 12 / 2013)$.

23 "no existía otra empresa en Chile con un acuerdo similar de un precio fijo por 15 años que está por debajo del de mercado. Y menos por debajo del precio del costo marginal que hoy se encuentra en US\$1.65 la libra o del costo total que llega a US\$2.33 la libra". Radic, S. El mercurio (15/12/2013).

24 De acuerdo a las Memorias Anuales de Codelco, año 2014, 2013, 2011 y 2010.

25 Cabe destacar que ninguno de los Reportes de Sustentabilidad emitidos a partir del 2010 por Codelco, fecha de la primera liquidación del SII, hace referencia a esta controversia entre la Corporación y el SII, como si ésta no fuera un elemento atañente a la Responsabilidad Social Empresarial de la Corporación.

26 Esta pérdida involucra sólo pérdida reales por contrato de cobertura de precio tomados para garantizar el precio a la Filial CuPIC, ello no incluye los que ha dejado de percibir Codelco por el diferencial de precio en el contrato de suministro de 836.250 toneladas, "fueron US $\$ 4.700$ millones que se giraron hacia los mercados financieros y el dueño, el Estado de Chile, es US $\$ 4.700$ millones más pobre que antes [...] pero aún nos queda el contrato de venta de cobre de Minmetals [...] sumando ambos son US\$7.000 millones". Tagle, A. (2014, 06, 04) www.emol.com. [on line]. Disponible en: http:/ / www.emol.com/noticias/economia/2014/06/01/663039/ ex-director-de-codelco-denuncia-perdidas-de-us-4700-millones-por-contratos-a-futuro-firmados-en-2005.html $(31 / 07 / 2015)$

27 Pulso (2015, 06, 02) www.punto.cl [on line]. Disponible en: http:/ / static.pulso.cl/20150601/2126575.pdf (31/07/2015). 
concretizó a través de Ley 20.392.

En el periodo 2005-2006 en donde la Corporación selló la transacción con Minmetals, el GC era bastante particular. El Directorio no era eminentemente profesional, en él podíamos encontrar a los ministros de Minería y Hacienda y a un representante de las Fuerzas Armadas. A este directorio no se les aplicaban las normas sobre derechos, obligaciones, responsabilidades y prohibiciones aplicables a las empresas privadas según la Ley de Sociedades Anónimas, lo cual justificó en cierta medida que la Comisión Investigadora no declarara responsables de lo que catalogó como "un pésimo negocio".

De acuerdo a Bernal et al. (2012), las modificaciones al GC introdujo la adopción de un Código de Ética y Valores, además de un fortalecimiento de los temas de seguridad, ambiental y responsabilidad social. Se estableció igualmente un proceso de nominación independiente y técnico del Ejecutivo Principal (CEO) así como un proceso de renovación del equipo de Alta Gerencia decuplado de los cambios de Gobierno. Además se permitió la capitalización de utilidades USD 376M (20\% de la utilidad neta).

Tabla I. Cambios en el Gobierno Corporativo de Codelco.

\begin{tabular}{|c|c|c|}
\hline $\begin{array}{l}\text { SOBRE EL } \\
\text { DIRECTORIO }\end{array}$ & ANTES & DESPUÉS \\
\hline $\begin{array}{l}\text { Estructura del } \\
\text { Directorio }\end{array}$ & $\begin{array}{l}7 \text { directores: } \\
\checkmark \text { Ministro de Minas (Presidente del } \\
\text { Directorio) } \\
\checkmark \text { Ministro de Finanzas } \\
\checkmark 2 \text { representantes del Presidente } \\
\text { de la República } \\
\checkmark 1 \text { representante de las Fuerzas } \\
\quad \text { Armadas } \\
\checkmark 2 \text { representantes sindicales }\end{array}$ & $\begin{array}{l}9 \text { directores: } \\
\checkmark 4 \text { directores seleccionados por el } \\
\text { Consejo Gerencial Público } \\
\checkmark 3 \text { Representantes del Presidente } \\
\text { de la República } \\
\checkmark 2 \text { Representantes sindicales }\end{array}$ \\
\hline Término & Periodo Presidencial & $\begin{array}{l}4 \text { años rotando parcialmente } \\
\text { (escalonado) }\end{array}$ \\
\hline $\begin{array}{l}\text { Roles y } \\
\text { Responsabilidades }\end{array}$ & $\begin{array}{l}\checkmark \text { Establecer políticas generales } \\
\checkmark \text { Aprobar inversiones por encima } \\
\text { de US\$ } 50 \text { millones } \\
\checkmark \text { No responsabilidad (civil y/o } \\
\text { penal) } \\
\checkmark \text { No regulado por el Derecho } \\
\quad \text { Societario }\end{array}$ & $\begin{array}{l}\checkmark \text { Nomina y designa al Presidente } \\
\text { Ejecutivo } \\
\checkmark \text { Aprueba el Plan Estratégico } \\
\checkmark \text { Responsabilidades civiles y } \\
\text { penales por sus decisiones } \\
\checkmark \text { Regidos por el derecho societario }\end{array}$ \\
\hline
\end{tabular}

Fuente: Extraído de Bernal et al. (2012, p. 42).

En teoría, y cumpliendo en acciones lo que estas modificaciones significan, el nuevo GC de Codelco podría evitar en el futuro, episodios como los observados en el caso Codelco-Minmetals, considerando además que la responsabilidad civil y penal en las decisiones que toma el directorio vendría a desincentivar comportamientos en los cuales se aprueben montajes financieros-tributarios que pueden ser catalogados como mecanismos de evasión y/o elusión fiscal.

\section{CONCLUSIONES}

Este estudio ha permitido establecer que existiría una relación entre el concepto de GC utilizado en una empresa con la forma en que se definen conceptualmente los impuestos. De esta forma, dado que en Chile se emplea un concepto tradicional de GC, se constata que los contribuyentes chilenos perciben a los impuestos como una carga que debe ser evitada, utilizando diversos mecanismos. 
Además, se han planteado algunos enfoques de los conceptos de planificación tributaria, elusión y evasión. Es posible establecer que el concepto de planificación tributaria correspondería a elusión sin dolo, pero también existiría elusión con dolo y evasión que sería una actividad ilegal.

Dada la importancia de la recaudación fiscal, vía impuestos, para la distribución de ingresos y su impacto en la desigualdad social y la disminución de la pobreza, cada vez existen más partidarios a nivel mundial de que el concepto de GC evolucione hacia un enfoque pluralista que refuerce el cumplimiento de las obligaciones fiscales como un medio de contribución al capital social.

En lo relacionado a los estudios de casos logramos ilustrar situaciones de elusión y evasión fiscal, de gran envergadura, en dos tipos de empresas, una privada y la otra pública.

En ambos casos podemos observar la presencia de lo que Sikka (2010) denominó “hipocresía organizada" entre el discurso público y las acciones concretas. De hecho, los efectos negativos de una "persistencia de la hipocresía organizada es una espada de doble filo y puede convertirse en un pasivo [...] Las tensiones pueden permanecer ocultas, pero siempre existe la posibilidad de que los empleados descontentos, ONG, periodistas de investigación, denunciantes y poderes del Estado pueden exponer las contradicciones y proporcionar la publicidad negativa que lleva a la pérdida de la legitimidad y de nuevos ingresos" (Sikka, 2010, p. 157, trad. propia).

Prueba de lo anterior, es la situación delicada que vive actualmente el Grupo Penta, en donde parte importante de sus activos han sido liquidados luego de hacerse pública la situación de evasión fiscal y financiamiento a campañas políticas. En el caso de Codelco, la situación fue considerada de carácter reservado, por lo tanto el impacto en los riesgos asociados a la reputación se han tratado de minimizar.

Postulamos que la orientación de los GC hacia la sola maximización de los beneficios para los accionistas tiene por consecuencia considerar los impuestos de las empresas como meros gastos que deben ser reducidos al máximo, ello se potencia cuando los altos ejecutivos son remunerados en función de los resultados financieros que generan, "el desarrollo de remuneración por rendimiento se ha visto acompañada por la intensificación de una industria que asesora a las empresas sobre las estrategias para evitar los impuestos. Contadores, abogados y expertos en servicios financieros no sólo proporcionan nuevas interpretaciones de la ley y las habilidades técnicas para permitir a algunos a evitar los impuestos, también legitiman vocabularios y discursos que tratan de normalizar la evasión de impuestos" (Sikka, 2010, p. 156, trad. propia).

Lo anterior se ve reflejado en los asesores financieros involucrados en la operación entre Codelco y Minmetals que proponen y validan estructuras tributarias que tienen por consecuencia deslocalizar los resultados.

El caso de Codelco-Minmentals, sólo ilustra lo que se practica cotidianamente en las Industrias Extractivas, "la aparición de la producción mundial crea nuevas y amplias oportunidades para las estrategias de precios de transferencia, permitiendo a las empresas trasladar beneficios a los lugares más deseables y evitar así impuestos" (Sikka \& Willmott, 2010, p. 345, trad. propia). La aparición de microestados conocidos como Paraísos Fiscales, los cuales ofrecen un tratamiento fiscal ventajoso y aseguran el secreto de los negocios no hace más que potenciar las prácticas elusivas de impuesto. En este contexto es comprensible que la postura de SII fuera "que no puede dejar que una empresa estatal haga a vista y paciencia de todos, lo que está estrictamente prohibido a las privadas" (El mercurio, 15/12/2013).

Todo lo anteriormente expuesto nos lleva a reflexionar sobre el rol social de la contabilidad y sobre la "necesidad de ir más allá de la imagen corporativa cuidadosamente cultivada y comprometerse con prácticas corporativas actuales y considerar su impacto en las vidas de las personas" (Sikka, 2010, p. 166, trad. propia), las pérdidas en los ingresos del Estado presentadas en estos dos estudios de casos, no sólo representan una pérdida material en la capacidad que tiene éste para responder a las necesidades de la población chilena, como lo son la salud y la educación, sino también refleja una pérdida de la confianza de los chilenos y los inversionistas actuales y potenciales en el Estado y sus Instituciones.

Este estudio es un aporte a la literatura contable en donde como lo exponen Sikka \& Willmott, (2010, p. 353, trad. propia) "hay un silencio ensordecedor sobre la participación de la técnica contable en la ampliación de las desigualdades sociales y la limitación de los recursos disponibles para los bienes públicos".

\section{BIBLIOGRAFÍA}

ACFE (2014). Report to the nations on occupational fraud and abuse [online]. Disponible en: http:/ / www.acfe.com/rttn-download-2014. $\operatorname{aspx}(20 / 06 / 2015)$. 
Bernal, A., Oneto, A., Penfold, M., Schneider, L. \& Wilcox, J. (2012). Gobierno Corporativo en América Latina. Importancia para las empresas de propiedad estatal. Serie Políticas Públicas y Transformación Productiva, 6, 40-43.

Capron, M. (2005). Les enjeux de la mise en œuvre des normes comptables internationales. En Les normes comptables internationales instruments du capitalisme financier (p. 5-23). Paris : Édition La découverte.

Charreaux, G. \& Desbrières, P. (1998). Gouvernance des entreprises: valeur partenariale contre valeur actionnariale. Finance Contrôle Stratégie, 1 (2), 57-88.

Comisión Especial Investigadora (2014). Informe de la comisión especial investigadora encargada de analizar los contratos, operaciones y forwards realizados por Codelco entre los años 2005 y 2007 [online]. Disponible en: http://www.camara. $\mathrm{cl} /$ pdf.aspx?prmTIPO=MANDATOSGRALDET \& prmID $=23903 \&$ prm TIPODOC $=$ LO CAL\&prmPERIODO=2014-2018 (30/07/2015).

Jiménez, J. P., Gómez, J. C., Podestá, A., (2010). Evasión y equidad en América Latina. Naciones Unidas, Comisión Económica para América Latina y el Caribe (CEPAL), Deutsche Gesellschaft für Technische Zusammenarbeit (GTZ), Ministerio Federal de Cooperación Económica y Desarrollo de Alemania (BMZ).

Jorratt, M. (2013). Gastos tributarios y evasión tributaria en Chile: evaluación y propuestas. En Tributación para el desarrollo. Estudios para la reforma del sistema chileno (39-142) [online]. Disponible en: http://www.cepchile.cl/PDFs_ CEP/Libro_Tributacion-para-el-desarrollo.pdf (17/04/2015).

Orellana, C., Azúa, D. (2014). Gobierno corporativo, transparencia e información: Un análisis teórico y un estudio de caso. Capic Review, 12 (1), 27-41.

Oxfam. (2014). Justicia fiscal para reducir la desigualdad en Latinoamérica y el Caribe [online]. Disponible en https://www.oxfam.org/sites/ www.oxfam.org/files/file_attachments/justicia_fiscal_para_reducir_la_desigualdad_en_latinoamerica_y_el_caribe_.pdf (10-07-2015).
Richard, J. (2009). Comptabilité environnementales. En Encyclopédie de comptabilité, contrôle de gestion et audit (489-501). Paris: Édition Economica.

Richard, J., Collette, C., Bensadon, D. \& Jaudet, N. (2011). Comptabilité Financière: Système comptable français et normes IFRS. Paris: Dunod.

Rivas, N. y Paillacar, C. (2007). Caracterización de la elusión fiscal en el impuesto a la renta de Chile. Capic Review, 5, 19-32.

Sikka, P. (2010). Smoke and mirrors: Corporate social responsibility and tax avoidance. Accounting Forum, 34 (3-4), 153-168.

Sikka, P. \& Willmott, W (2010). The dark side of transfer pricing: Its role in tax avoidance and wealth retentiveness. Critical Perspectives on Accounting, 21 (4), 342-356.

Tanzi, V. (2001). La globalización y la acción de las termitas fiscales. Finanzas $\&$ Desarrollo, 38 (1), 34-37.

Van Weezel, A. (2007). Delitos Tributarios. Santiago de Chile. Editorial Jurídica de Chile.

Yáñez, J. (2015). Tributación: Equidad y/o Eficiencia. Revista de Estudios Tributarios, 12, 223259. 